\title{
Nanoscale
}

CORRECTION

Cite this: Nanoscale, 2017, 9, 4310

DOI: 10.1039/c7nr90051a

rsc.li/nanoscale

\section{Correction: Nanoparticle-based photoacoustic analysis for highly sensitive lateral flow assays}

Yunfei Zhao, ${ }^{a}$ Yin Huang, ${ }^{a}$ Xiangwei Zhao, ${ }^{* b}$ John F. McClelland ${ }^{c}$ and Meng Lu*a,d

Correction for 'Nanoparticle-based photoacoustic analysis for highly sensitive lateral flow assays' by Yunfei Zhao et al., Nanoscale, 2016, 8, 19204-19210.

In the original manuscript, the e-mail address of Xiangwei Zhao was displayed incorrectly. The correct e-mail address is xwzhao@seu.edu.cn and the complete, corrected affiliation and contact details are presented herein.

The Royal Society of Chemistry apologises for these errors and any consequent inconvenience to authors and readers.

\footnotetext{
${ }^{a}$ Department of Electrical and Computer Engineering, Iowa State University, Ames, Iowa 50011, USA. E-mail: menglu@iastate.edu ${ }^{b}$ State Key Laboratory of Bioelectronics, Southeast University, Nanjing, Jiangsu 211189, P. R. China. E-mail: xwzhao@seu.edu.cn ${ }^{c}$ MTEC Photoacoustics Inc., 3507 Oakland Street, Ames, IA 50014, USA

${ }^{d}$ Department of Mechanical Engineering, Iowa State University, Ames, Iowa 50011, USA
} 